\title{
L'amministrazione dei centri minori della regione di Umma nel periodo protodinastico
}

\author{
( Armando Bramanti \\ Consejo Superior de Investigaciones Científicas, Madrid, España ${ }^{1}$
}

Fecha de recepción: 25 de julio de 2021. Fecha de aceptación: 18 de agosto de 2021.

\begin{abstract}
La administración de los centros menores de la región de Umma en el periodo protodinástico
\end{abstract}

\begin{abstract}
Resumen
Tras una breve introducción al concepto de geografía histórica y a su aplicación a los estudios del Próximo Oriente antiguo se presentan en este ensayo algunas reflexiones de tipo histórico-geográfico acerca de los centros menores de la región de Umma en el periodo protodinástico. Se analiza en particular la información conocida en relación con los centros de Zabala, Kian, Dulum y Kardahi en la época de referencia, probando la importancia de una aproximación histórico-geográfica en la reconstrucción de un complejo panorama económico-administrativo resultante en múltiples niveles jerárquicos.
\end{abstract}

Palabras clave: Umma, periodo protodinástico, centros menores, geografía histórica, administración

1 L'autore è grato agli editori del presente dossier "Ciudades y urbanismo en el mundo antiguo" per l'invito a partecipare ed in questo modo sviluppare alcuni dei temi trattati in una serie di lezioni tenute in Argentina presso l'Instituto de Historia Antigua Oriental (IHAO) della Universidad de Buenos Aires (22/05/2019) e la Universidad Nacional de Rosario (23/05/2019). Tali lezioni hanno fornito un'introduzione alla geografia storica della Mesopotamia del terzo millennio a.e.c., inserendosi in un più ampio progetto di collaborazione di ricerca e didattica che ha visto l'autore impegnato in vari centri argentini, tra cui la UBA (Buenos Aires), la UNR (Rosario) e la UNL (Santa Fe) (cfr. Bramanti et al., 2021 e Luciani et al., in preparazione). Il tema della geografia storica della regione di Umma è stato sviluppato anche durante un soggiorno di ricerca presso l'Istituto Svizzero di Roma (ISR) nell'AA 2019/2020, istituzione verso la quale si esprime gratitudine anche per il supporto logistico offerto durante un annus horribilis che ha segnato particolarmente non solo le nostre vite ma anche la produzione scientifica a livello globale. 


\title{
The Administration of Minor Centers in the Early Dynastic Umma Region
}

\begin{abstract}
After a brief introduction to the concept of historical geography and to its application to Ancient Near Eastern Studies, this article presents some thoughts on the historical geography of minor centers in the Early Dynastic Umma region. More specifically, the author analyzes the so far known information about the centers of Zabala, Ki'an, Dulum, and Kardahi during the reference time period, proving the importance of a historical geographical approach in the reconstruction of a complex, multi-layered economic and administrative panorama.
\end{abstract}

Keywords: Umma, Early Dynastic period, minor centers, historical geography, administration

\section{La geografia storica ed il Vicino Oriente antico}

La geografia storica è una disciplina dallo sviluppo relativamente recente che ha come obiettivo lo studio dei fenomeni geografici in relazione allo scorrere del tempo e dunque alla storia. Nel 1975 la fondazione in Inghilterra del Journal of Historical Geography ha aperto un dialogo internazionale circa l'orizzonte di riferimento della disciplina. Gli editori di questa prima rivista specializzata si sforzavano allora di mantenere tale orizzonte il più aperto e generico possibile, accettando contributi da "scholars of any disciplinary provenance who have something to say about matters of geographical interest relating to past time". Dal 1969 al 1993 la Deutsche Forschungsgemeinschaft (DFG) ha finanziato in Germania l'ambizioso progetto del Tübinger Atlas des Vorderen Orients (TAVO), un dettagliato atlante in 296 tavole che ha segnato l'inizio di un nuovo capitolo nella comprensione della geografia del Vicino Oriente antico. Questo progetto ha reso possibile a partire dal 1974 la pubblicazione di numerosi volumi del Répertoire Géographique des Textes Cunéiformes (RGTC) come frutto della rielaborazione dei dati contenuti nelle tavole del TAVO (cfr. e.g. RGTC 2, VIII) e della raccolta ed interpretazione dei toponimi dei testi cuneiformi relativi alle varie epoche affrontate dai singoli volumi.

L'esistenza di questi primi, fondamentali strumenti di lavoro ha incentivato negli ultimi decenni la ricerca e l'apparizione di importanti pubblicazioni nell'ambito della geografia storica del Vicino Oriente antico. È questo il caso di atti di convegni internazionali (e.g. Liverani, 1995, e Milano et al., 1999-2000), volumi collettivi come risultato di collaborazioni in progetti di ricerca (e.g. CancikKirschbaum e Ziegler, 2009 e 2014) ed anche singole monografie (e.g. Gander, 2010, e Barjamovic, 2011) che hanno fornito le linee guida per lo sviluppo di un campo necessariamente multidisciplinare, nel quale la collaborazione di discipline quali la storia, la geografia, l’archeologia e la filologia non è solo auspicabile ma anche inevitabile. 
Di particolare interesse il lavoro compiuto nell'ambito del progetto internazionale ARCANE (Associated Regional Chronologies for the Ancient Near East), che illustra perfettamente come lo studio delle cronologie non possa prescindere da una revisione delle conoscenze geografiche relative ad una determinata facies storico-culturale e dunque da un'ottica storico-geografica. All'interno di questo progetto ed in merito alla geografia storica del terzo millennio contributi come quelli di H. Steible (2015) e M. G. Biga (2015), rispettivamente per le regioni di Šuruppak ed Ebla, sono illuminanti poiché ribadiscono l’importanza dello studio dei processi amministrativi e culturali nell'ottica di una corretta ricostruzione storico-geografica, trascendendo la mera collezione di toponimi ed elevando la disciplina verso un nuovo livello. ${ }^{3}$

\section{L'orizzonte geografico di Umma nel periodo protodinastico}

\subsection{Il corpus}

In questo articolo si presenteranno, senza pretesa di completezza, alcune riflessioni circa i centri minori della regione di Umma durante il periodo protodinastico e la loro amministrazione. Il corpus di testi in oggetto (noto in letteratura come Early Dynastic Umma corpus) è composto da circa 1100 tavolette di natura prevalentemente amministrativa. Un gran numero di questi testi si trova oggigiorno presso la Rosen Collection (Cornell University, Ithaca, NY), Schøyen Collection (Spikkestad, Norvegia), Banca d'Italia (Roma), e Yale Babylonian Collection (New Haven, CT). Il resto del corpus è sparpagliato in lotti più piccoli presso musei, università e collezioni private in vari paesi. Fino ad un paio di decenni fa il corpus protodinastico di Umma consisteva essenzialmente di poco più di un centinaio di testi identificati da M. A. Powell nel 1978. A seguito degli eventi causati della Prima Guerra del Golfo centinaia di nuovi documenti cuneiformi relativi alle epoche più antiche della storia mesopotamica sono stati portati all'attenzione della comunità assiriologica. Questo, unitamente ai recenti scavi archeologici dello Iraqi State Board of Antiquity and Heritage e, più recentemente, dello Slovak Archaeological and Historical Institute, ha fatto in modo che il corpus protodinastico di Umma raggiungesse l'ammontare di circa 1100 documenti. ${ }^{4}$ La magnitudine di questo corpus consentirà senzaltro in futuro studi approfonditi del contesto storico-geografico della regione di

3 A tal proposito si evidenzia l'urgenza di considerare in studi storico-geografici all'interno della categoria "toponimi" non soltanto i nomi di città, ma anche quelli di centri minori, campi e corsi d'acqua e di registrare la presenza di ogni elemento che possa in qualche modo mostrare una base geografica come edifici pubblici (templi, palazzi, granai) e privati (households ed abitazioni private), le cui reciproche interazioni amministrative molto possono suggerire circa la loro presenza sul territorio.

4 Per una lista parziale dei testi appartenenti a questo corpus, si veda Bramanti, 2017a: 33, n. 1 e, più recentemente, Notizia e Almamori, 2021, entrambi con letteratura precedente. 
Umma, così come ha consentito negli ultimi anni una migliore comprensione della sua amministrazione, prosopografia e paleografia. ${ }^{5}$

\section{2. Zabala}

Zabala è, secondo la nostra attuale comprensione, il centro minore più importante della regione di Umma e quello che ha restituito il maggior numero di documenti relativi all'epoca protodinastica. La sua identificazione con Tell Ibzeikh (no. 69 nella survey della regione di Uruk di R. McC. Adams e H. J. Nissen, 1972) non lascia dubbi grazie al ritrovamento in situ di alcuni mattoni impressi relativi alla costruzione dell'Ezikalamma di Inanna da parte di Hammurapi (RIME 4.3.6.15). ${ }^{6}$

Una gran parte dei documenti protodinastici provenienti da Zabala si riferiscono all'amministrazione del tempio di Inanna, soprattutto durante il lungo governatorato di Me’annedu (Bartash, 2017: 3-4; Bramanti, 2017a). La natura di questi documenti, relativi per la maggior parte alla gestione della terra e delle attività e prodotti agricoli, suggerisce come il tempio di Inanna di Zabala fosse responsabile per l'amministrazione locale ed in qualche modo indipendente dall'amministrazione centrale di Umma. Da Zabala proviene anche un archivio di 106 testi noto in letteratura come Archivio di Lugalzagesi, ad oggi l'unico archivio ricostruito del corpus protodinastico di Umma. ${ }^{7}$ All'interno di questo archivio un dossier di 26 documenti tratta la gestione di oggetti metallici come zappe, falcetti, lance, punte di lancia, calderoni ed alcune quantità di rame. Tre testi di questo dossier menzionano un palazzo (e $\mathbf{e}_{2}$-gal) $(B I N ~ 8,87 ; 88 ; 96)$. In un caso il palazzo elargisce i beni $\left(\mathbf{e}_{3}\right)$, mentre in altri due casi i beni sono portati a palazzo $\left(\mathbf{l a h}_{4 / 5}\right)$. Queste tre occorrenze mostrano i limiti dell'indipendenza dell'amministrazione locale del tempio di Inanna a Zabala, il quale si rimetteva allautorità centrale del palazzo nel caso della gestione di questi oggetti metallici. Poiché non abbiamo ragione di sostenere l'esistenza di un palazzo a Zabala o in altri centri minori della regione ${ }^{8}$ questo dossier documenta l'interazione tra l'amministrazione locale di Zabala e quella centrale di Umma.

\subsection{Ki'an}

Ki’an è nominata insieme alla città di Umma in un'iscrizione reale di Lugalzagesi (RIME 1.12.7). In entrambe le città il sovrano afferma di aver costruito un Tempio dello Scettro (e $\mathbf{e}_{2}$-⿳⺈idru), inserendo in questo modo la narrativa della

5 Solo a titolo di esempio si citano due recenti volumi di edizioni di testi (Notizia e Visicato, 2016, e Bartash, 2017) e due recenti studi su amministrazione (Bramanti, 2020) e paleografia (Bramanti, 2021).

6 Sull'identificazione di Zabala, si veda anche Molina, 2017: 171 con letteratura precedente. Ai suoi argomenti si può aggiungere la menzione di "Tel-el-Bouzekh" nella corrispondenza dell'antiquario E. S. David, che può essere indirettamente collegata all'acquisto da parte dell'università di Yale della maggior parte delle tavolette dell'archivio di Lugalzagesi di Zabala (Powell, 1976: 103 e Powell, 1978: 7-8).

7 Per un'edizione completa dell'Archivio di Lugalzagesi e per ulteriori considerazioni sull'amministrazione di Zabala si veda Bramanti, 2017b. 
costruzione di templi nel suo cursus honorum. Kian viene nominata anche nella più celebre iscrizione di Lugalzagesi RIME 1.14.20.1 in cui, insieme alle altre città del sud mesopotamico, questo centro urbano minore celebra l'ascesa del sovrano al trono di Uruk. L'identificazione di Kỉan con Tell Shmeit, già suggerita da Adams e Nissen nel 1972 (no. 168), è stata più recentemente suffragata a seguito di prospezioni e scavi presso Tell Shmeit (Abdul Rahim, 2003-2004: 201), sito interessato dall'attività archeologica dello Iraqi State Board of Antiquity and Heritage all'inizio degli anni ' 00.

Numerosi testi amministrativi all'interno del corpus protodinastico di Umma menzionano Kian ed i suoi abitanti, ma due in particolare meritano la nostra attenzione. BIN 8, 32 e 106, appartenenti al già menzionato dossier dei metalli dell'archivio di Lugalzagesi, sono le ricevute di due transazioni di falcetti e lance avvenute a Kian. Poiché il dossier in oggetto forma parte di un archivio proveniente dal tempio di Inanna a Zabala, è possibile dedurre che quest'ultimo si facesse carico non soltanto dell'amministrazione locale della città di Zabala ma anche di quella di alcuni centri minori nelle sue vicinanze come Kian che, a causa delle loro ridotte dimensioni, erano probabilmente sprovvisti di un vero e proprio centro amministrativo. Grazie a queste evidenze sporadiche è possibile dunque intravedere la complessa stratificazione dei meccanismi amministrativi nella regione di Umma, dove un'amministrazione centrale palatina delega la gestione degli affari locali ai templi ubicati nei centri minori, nei cui archivi afferisce inoltre la documentazione relativa ai villaggi circostanti.

\subsection{Dulum}

Dulum (lettura comune del composto NAGAAR.BU) è la sede della divinità Nin-Dulum nella composizione protodinastica nota come Inni Zame (inno no. 26, linee 91-96; cfr. Krebernik e Lisman, 2020: 117) ed è elencata subito dopo la città di Ereš, sede di Nisaba (cfr. anche Cavigneaux e Krebernik, 1998-2001, e Krebernik, 2017: 204). Già Powell aveva identificato Nin-Dulum come una divinità il cui culto aveva sede nella regione di Umma e più specificamente a Zabala sulla base delle evidenze protodinastiche e neosumeriche (1976: 102103). È stato suggerito che Dulum possa identificarsi con il sito di Tell Șalbūh (Frayne, 2007: 358-359 e 2009: 58, n. 85), non ancora esplorato archeologicamente. Questa identificazione è tuttavia dubbia poiché la survey della regione di Uruk ha mostrato per questo sito solo un'occupazione tardo-sasanide ed islamica incipiente (Adams e Nissen, 1972: 221, n. 36). Ad ogni modo, è impossibile allo stato attuale delle conoscenze proporre una diversa identificazione.

Notizia e Visicato suggeriscono che le attestazioni di Dulum nel corpus protodinastico di Umma possano riferirsi ad un non meglio identificato tempio di (Nin-) Dulum. In questo caso l'espressione ensi ${ }_{2}$ dulum $_{2}$ non si riferirebbe al sovrano della città ma alla figura principale del tempio di Dulum (Notizia e Visicato, 2016: 56, con una lista di attestazioni di questa espressione). Ciononostante, ed in aggiunta alla testimonianza fornita dagli Inni Zame, è preferibile interpretare Dulum come un centro urbano in base alle seguenti ragioni:

a) Dulum occorre insieme ad alcuni nomi di persona per identificare la provenienza degli individui associati a questo toponimo e risolvere dunque alcuni 
casi di omonimia (cfr. Bartash, 2013:49). Si veda, ad esempio, silim-utu dulum in CUSAS 23, 32 rev. i 4 e $\mathbf{U}_{2} \cdot \mathbf{U}_{2}$ dulum $_{2}$ in Powell, 1978, no. 13 ii 5 e no. 14 i 4 nell'archivio di Lugalzagesi;

b) l'esistenza di un campo associato a Dulum (a-ša dulum $_{2}$; Bartash, 2013: 224, sub voce, e Notizia e Visicato, 2016: 279, sub voce) ma mai associato direttamente alla divinità Nin-Dulum e dunque vincolato al suo tempio;

c) il testo inedito $\mathrm{P} 270822^{9}$ è un registro di appezzamenti di terreno del corpus protodinastico di Umma (cfr. Notizia e Visicato, 2016: 11) risalente all'epoca di Meannedu. In questo testo Dulum occorre dopo una linea in bianco e definisce la localizzazione geografica degli appezzamenti precedenti (rev. iii 4), assumendo la stessa funzione di Zabala, che occorre precedentemente nello stesso testo (rev. ii 8) - le altre categorie che definiscono gli appezzamenti in questo documento si riferiscono a nomi di persona o households.

Loccorrenza di un išib dulum 2 , "prete lamentatore di Dulum" (Notizia e Visicato, 2016: 273, sub voce) potrebbe indicare l'esistenza di un personale di culto del tempio di (Nin-)Dulum nella città di Dulum, ma non esclude l'esistenza di Dulum come centro urbano in sé. Nel caso si favorisse l'interpretazione di Dulum come nome geografico si dovrebbe postulare un'indipendenza almeno momentanea di questa città, la quale aveva, difatti, un suo governatore (ensi $\mathbf{~}_{2}$ ). I testi suggeriscono che Dulum avrebbe dunque mantenuto contatti amichevoli con Zabala (e quindi con Umma) durante il governatorato di Me'annedu, prima di essere sottomessa da Lugalzagesi durante una campagna militare precedente al settimo anno di governatorato di quest'ultimo.

\subsection{Kardahi}

Kardahi è un centro minore relativamente ben attestato nella documentazione neosumerica. Sue menzioni si ritrovano principalmente nella documentazione di Umma (nella grafia kar-da-hi ma soprattutto har-da-hi), sebbene attestazioni sporadiche si incontrino anche a Ĝirsu (kar-da-hui) ed a Garšana ( gar $_{3}$-dahi). I testi di Umma suggeriscono la presenza a Kardahi di un'amministrazione relativa ad un tempio di Nergal / Meslamta'ea, laddove i testi di Girsu e Garšana citano del personale (erin ${ }_{2}$ e ĝuruš) proveniente da questa città. ${ }^{10}$

Kardahi è solo sporadicamente attestata in testi sargonici (CUSAS 26, 163, e Robson e Zólyomi, 2014: 191 [Mesaĝ]) e protodinastici (Powell, 1978, no. 8 [Lugalzagesi]) della regione di Umma. Quest'ultimo, che riporta nel suo verso un registro di appezzamenti di terreno di Zabala, è, al meglio della conoscenza dellautore, la prima attestazione di questo toponimo (pace Robson e Zólyomi, 2014: 192), citato in questo caso come provenienza del prete purificatore (išib) ur-e $_{2}$ (rev. ii 2) (Fig. 1). Si noti che lo stesso prete appare anche in altri due documenti dello stesso archivio di Zabala, Powell, 1978 no. 10 e BIN 8, 114 (in

9 Accessibile online tramite questo numero di catalogo sul database Cuneiform Digital Library Initiative (CDLI) (https://cdli.ucla.edu/). 
quest'ultimo caso con il nome completo ur- $\mathbf{e}_{2}$-mah), suggerendo che Kardahi si trovasse in epoca protodinastica in una posizione di dipendenza amministrativa da Zabala, come già visto nel caso di Ki’an.
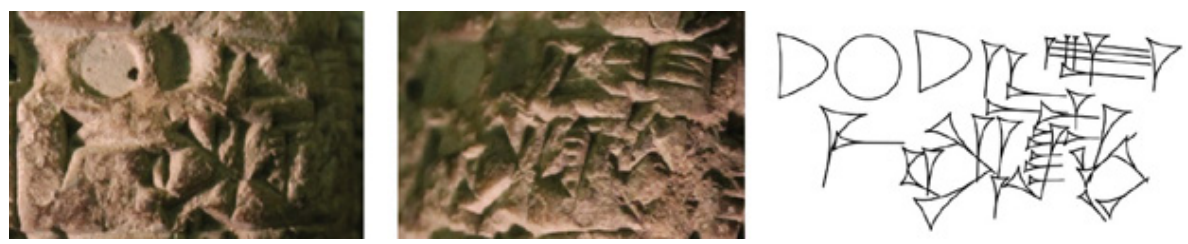

Fig. 1. Powell, 1978, no. 8 rev. ii 2 visto da due diverse angolazioni ed in copia. 1 (eše3) 1 (iku) ur-e $e_{2}$ išib kar-da-hi, “ 1 eše 1 iku (di terreno): Ure, il prete purificatore di Kardahi”.

L'esercizio scolastico di epoca sargonica CUSAS 26, 163, sembrerebbe invece fornire un indizio circa la localizzazione relativa di Kardahi. Dopo un elenco di nomi di persona inusuali nel recto, il testo riporta nel verso due toponimi, Kardahi e Ki’an (rev. 1-2). Questo lascerebbe intuire la vicinanza geografica dei due centri, citati per questo consecutivamente dall'apprendista scriba. ${ }^{11}$

\section{Conclusioni}

Nelle pagine precedenti si è presentato un panorama delle informazioni note ad oggi circa l'amministrazione dei quattro centri minori della regione di Umma attestati nella documentazione protodinastica: Zabala, Kian, Dulum e Kardahi. ${ }^{12} \mathrm{Ne}$ è risultato un quadro amministrativo complesso, con una gerarchia a più livelli il cui vertice è costituito dalla capitale Umma e dal suo palazzo che controlla a livello regionale il movimento di alcuni beni. Quest'ultimo delega ai templi dei centri minori l'amministrazione degli affari locali come la gestione della terra e delle messi - è questo il caso del tempio di Inanna a Zabala (e forse di Dulum?) - i quali si fanno carico anche dell'amministrazione di quei villaggi come Kian e Kardahi che, probabilmente a causa delle loro limitate dimensioni, non possedevano un proprio centro gestionale.

Queste considerazioni dimostrano inoltre l'utilità di un approccio storicogeografico nella ricostruzione dei processi amministrativi, e dunque nella comprensione della storia economica e sociale, a partire dalle fasi più antiche della documentazione mesopotamica.

11 Sull'interpretazione di CUSAS 26, 163 si veda anche Kraus, 2020: 18-19 e 71-72.

12 Si noti che la città di Apisal (a-pi $\left.\mathbf{~}_{\mathbf{4}}-\mathbf{s a l}_{\mathbf{4}}\right)$, importante centro urbano della provincia neosumerica di Umma, è attestata nei testi soltanto a partire dall'epoca sargonica (pace Schrakamp, 2015: 218; cfr. Westenholz, 2014: 129). Sull'identificazione di Apisal si veda Steinkeller, 2001: 54-56 e passim. Circa la possibilità dell'interpretazione come Apisal del segno composto $\mathbf{A B \times S ̌ \mathbf { S } _ { 2 }}$ dell'inno Zame no. 29 (linee 101-102) immediatamente precedente l'inno di Ĝišša / Umma, si veda Krebernik e Lisman, 2020: 118-119 con letteratura precedente. 


\section{Q Bibliografia}

"Abdul Rahim, M. S. (2003-2004). Prospecting in Site Shmeet 2001-2002, en: Sumer 52: 201-241. [in arabo]

» Adams, R. McC. e Nissen, H. J. (1972). The Uruk Countryside: The Natural Setting of Urban Societies. Chicago: University of Chicago Press.

»Barjamovic, G. (2011). A Historical Geography of Anatolia in the Old Assyrian Colony Period (Carsten Niebuhr Institute Publications 38). Copenhagen: Museum Tusculanum Press.

"Bartash, V. (2013). Miscellaneous Early Dynastic and Sargonic Texts in the Cornell University Collections (Cornell University Studies in Assyriology and Sumerology 23). Bethesda, MA: CDL Press.

"Bartash, V. (2017). Sumerian Administrative and Legal Documents ca. 2900-2200 $B C$ in the Schøyen Collection (Cornell University Studies in Assyriology and Sumerology 35). Bethesda, MA: CDL Press.

»Biga, M. G. (2015). The Geographical Scope of Ebla: Commerce and Wars. Some Remarks, en: Sallaberger, W. e Schrakamp, I. (eds.), Historical and Epigraphic Data for a Chronology of Mesopotamia in the Third Millennium B.C. (Associated Regional Chronologies for the Ancient Near East 3). Turnhout: Brepols, 181-190.

»Bramanti, A. (2017a). Three Administrative Texts from the Time of Me'annedu, en: Journal of Cuneiform Studies 69: 33-47.

"Bramanti, A. (2017b). Land Management in the Early Dynastic Umma Region: The Case of the Archive of Lugalzagesi (tesi di dottorato). Roma e Jena: Sapienza Università di Roma e Friedrich-Schiller-Universität Jena.

»Bramanti, A. (2020). Before the Harvest? Land-grain Accounts in Early Dynastic Umma, en: Borrelli, N. e Scazzosi, G. (eds.), After the Harvest. Storage Practices and Food Processing in Bronze Age Mesopotamia (Subartu 43). Turnhout: Brepols, 37-44.

"Bramanti, A. (2021). The Making of a Sign List: Toward a Paleography of Early Dynastic Umma, en: Bramanti, A., Kraus, N. e Notizia, P. (eds.), Current Research in Early Mesopotamian Studies. Workshop organized at the $65^{\text {th }}$ Rencontre Assyriologique Internationale, Paris 2019 (dubsar 21). Münster: Zaphon, 205-212.

"Bramanti, A., Luciani, F., Rovira, L., Gonçalves, C. e Chávez Fajardo, S. (2021). Conversaciones sobre Asiriología y enseñanza. Europa y América Latina: Internacionalizar, cooperar, descentralizar, en: Rodríguez, R. R. e Magneres, M. (eds.), Sociedades Antiguas del Mediterráneo y América: aproximaciones desde el Sur. Actas de Jornadas Patagónicas de Estudios sobre Sociedades Antiguas (UNPAUASJ) / I Jornadas Virtuales e Internacionales de Historia Antigua. Aportes Teóricos y Metodológicos en Tiempos de Pandemia (UNICEN). Buenos Aires: El Búho Desplumado, 331-364.

"Cancik-Kirschbaum, E. e Ziegler, N. (eds.) (2009). Entre les fleuves - I. Untersuchungen zur historischen Geographie Obermesopotamiens im 2.Jahrtausend v. Chr. (Berliner Beiträge zum Vorderen Orient 20). Gladbeck: PeWe-Verlag.

»Cancik-Kirschbaum, E. e Ziegler, N. (eds.) (2014). Entre les Fleuves - II. D’Aššur à Mari et au-delà (Berliner Beiträge zum Vorderen Orient 24). Gladbeck: PeWe- 
Verlag.

»Cavigneaux, A. e Krebernik, M. (1998-2001). Nin-duluma, en: Reallexikon der Assyriologie und Vorderasiatischen Archäologie 9: 340-341.

» Frayne, D. R. (2007). Presargonic Period (2700-2350 BC) (The Royal Inscriptions of Mesopotamia. Early Periods 1). Toronto-Buffalo-London: University of Toronto Press.

» Frayne, D. R. (2009). The Struggle for Hegemony in "Early Dynastic II" Sumer, en: The Canadian Society for Mesopotamian Studies, Journal 4: 37-75.

"Gander, M. (2010). Die geographischen Beziehungen der Lukka-Länder (Texte der Hethiter. Philologische und historische Studien zur Altanatolistik 27). Heidelberg: Universitätsverlag Winter.

" Kraus, N. L. (2020). Scribal Education in the Sargonic Period (Harvard Semitic Studies 67). Leiden-Boston: Brill.

»Krebernik, M. (2017). Zame/i-Hymnen, en: Reallexikon der Assyriologie und Vorderasiatischen Archäologie 15, 203-205.

" Krebernik, M. e Lisman, J. J. W. (2020). The Sumerian Zame Hymns from Tell Abū Șaläbīh (dubsar 12). Münster: Zaphon.

"Liverani, M. (ed.) (1995). Neo-Assyrian Geography (Quaderni di Geografia Storica 5). Roma: Università di Roma “La Sapienza”.

» Luciani, F., Rovira, L., Bramanti, A. e Milevski, I. (in preparazione). Arqueología y filología. El desafío de la construcción metodológica en las cátedras de Prehistoria General y Sociedades del Cercano Oriente (titolo provvisorio).

" Milano, L., De Martino, S., Fales, F. M. e Lanfranchi, G. B. (eds.) (1999-2000). Landscapes. Territories, Frontiers and Horizons in the Ancient Near East. Papers presented to the XLIV Rencontre Assyriologique Internationale. Venezia, 7-11 July 1997. Part I, Part II, Part III (History of the Ancient Near East / Monographs III/1, III/2, III/3). Padova: Sargon.

"Molina, M. (2017). Zabala/u(m), en: Reallexikon der Assyriologie und Vorderasiatischen Archäologie 15: 170-174.

»Monaco, S. F. (2011). Early Dynastic mu-iti Cereal Texts in the Cornell University Cuneiform Collections (Cornell University Studies in Assyriology and Sumerology 14). Bethesda, MD: CDL Press.

» Notizia, P. e Almamori, H. O. (2021). The City-State of Umma in the Pre-Sargonic Period. New Tablets from the Iraq Museum, en: Akkadica 142: 1-30.

"Notizia, P. e Visicato, G. (2016). Early Dynastic and Early Sargonic Administrative Texts Mainly from the Umma Region in the Cornell University Cuneiform Collections (Cornell University Studies in Assyriology and Sumerology 33). Bethesda, MA: CDL Press.

"Powell, M. A. (1976). Evidence for Local Cults at Presargonic Zabala, en: Orientalia Nova Series 45: 100-104.

»Powell, M. A. (1978). Texts from the Time of Lugalzagesi. Problems and Perspectives in Their Interpretation, en: Hebrew Union College Annual 49: 1-58.

» Robson, E. e Zólyomi, G. (2014). Mesag Reports a Murder: Cuneiform Tablets in the Collections of Norwich Castle Museum and Cambridge University Library, en: Iraq 76: 189-203. 
"Schrakamp, I. (2015). Geographical Horizons in Presargonic and Sargonic Archives, en: Sallaberger, W. e Schrakamp, I. (eds.), Historical and Epigraphic Data for a Chronology of Mesopotamia in the Third Millennium B.C. (Associated Regional Chronologies for the Ancient Near East 3). Turnhout: Brepols, 197-270.

»Steible, H. (2015). The Geographical Horizon of the Texts from Fara/Shuruppag, en: Sallaberger, W. e Schrakamp, I. (eds.), Historical and Epigraphic Data for a Chronology of Mesopotamia in the Third Millennium B.C. (Associated Regional Chronologies for the Ancient Near East 3). Turnhout: Brepols, 157-161.

»Steinkeller, P. (2001). New Light on the Hydrology and Topography of Southern Babylonia in the Third Millennium, en: Zeitschrift für Assyriologie und Vorderasiatische Archäologie 91: 22-84.

»Steinkeller, P. (2013). Corvée Labor in Ur III Times, en: Garfinkle, S. J. e Molina, M. (eds.), From the 21st Century B.C. to the 21st Century A.D. Proceedings of the International Conference on Neo-Sumerian Studies Held in Madrid, 22-24 July 2010. Winona Lake, IN: Eisenbrauns, 347-424.

"Westenholz, A. (2014). A Third-Millennium Miscellany of Cuneiform Texts (Cornell University Studies in Assyriology and Sumerology 26). Bethesda, MA: CDL Press. 